Physics

Physics Research Publications

\title{
Change in speed and direction of the jet near the core in the quasar 3C 279
}
S. G. Jorstad*
A. P. Marscher ${ }^{\dagger}$
M. L. Lister
A. M. Stirling**
T. V. Cawthorne ${ }^{\dagger \dagger}$
J. L. Gomez
W. K. Gear ${ }^{\S}$

http://docs.lib.purdue.edu/physics_articles/366 


\title{
CHANGE IN SPEED AND DIRECTION OF THE JET NEAR THE CORE IN THE QUASAR 3C 279

\author{
Svetlana G. Jorstad, ${ }^{1,2}$ Alan P. Marscher, ${ }^{1}$ Matthew L. Lister, ${ }^{3}$ Alastair M. Stirling, ${ }^{4,5}$ \\ Timothy V. Cawthorne, ${ }^{4}$ José-Luis Gómez, ${ }^{6,7}$ and Walter K. Gear ${ }^{8}$ \\ Received 2004 January 5; accepted 2004 March 9
}

\begin{abstract}
Intensive monitoring of the innermost jet in the quasar 3C 279 at $43 \mathrm{GHz}$ from 1998 March to 2001 April with the Very Long Baseline Array, in combination with previous observations, reveal (1) an increase in apparent speed from $5 c$ to $17 c$ and (2) a change in projected direction of the jet by $\sim 20^{\circ}$. These effects could result from a small increase in the intrinsic viewing angle of the jet nozzle from $\Theta \lesssim 0.5$ to $\Theta \lesssim 1^{\circ}$. 6 , with no increase required in the actual speed of the flow, which corresponds to a Lorentz factor $\gamma \gtrsim 20$. The Lorentz factor $\sim 20$ and viewing angle $\sim 0.5$ imply that the Doppler factor is at least 39 close to the core; this extreme value explains the high flux density and rapid variability of 3C 279 across the electromagnetic spectrum.
\end{abstract}

Key words: galaxies: jets - quasars: individual (3C 279)

\section{INTRODUCTION}

The quasar $3 \mathrm{C} 279$, one of the brightest sources in the radio sky, has a radio jet featuring highly superluminal motion of bright knots (components) that appear to be ejected from a presumably stationary "core." The apparent speed ( $\beta_{\text {app }}$, in units of $c$ ) of a component is a function of the bulk Lorentz factor $\gamma$ and the viewing angle $\Theta$ :

$$
\beta_{\mathrm{app}}=\frac{\sqrt{\gamma^{2}-1} \sin \Theta}{\gamma-\sqrt{\gamma^{2}-1} \cos \Theta} .
$$

The first rapid variability in the milliarcsecond scale structure of the quasar was observed by Knight et al. (1971). Since then, at least nine superluminal components have been detected between 1970 and 1997. These knots cover a wide range of

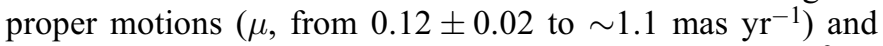
projected position angles $\left(\Phi\right.$, from approximately $-120^{\circ}$ to approximately $-150^{\circ}$ ) within 2 mas of the core (Cotton et al. 1979; Pauliny-Toth et al. 1981; Unwin et al. 1989; Wehrle et al. 2001, hereafter W01; Jorstad et al. 2001a, hereafter J01). Here we report an increase in apparent speed and a swing in position angle that occurred near the core of the quasar between 1997 and 1998, which we have traced through bimonthly observations with the Very Long Baseline Array (VLBA).

\footnotetext{
${ }^{1}$ Institute for Astrophysical Research, Boston University, 725 Commonwealth Avenue, Boston, MA 02215-1401; jorstad@bu.edu, marscher@bu.edu.

2 Sobolev Astronomical Institute, St. Petersburg State University, Universitetskij pr. 28, 198504 St. Petersburg, Russia.

3 Department of Physics, Purdue University, 1396 Physics Building, West Lafayette, IN 47907-1396; mlister@physics.purdue.edu.

${ }^{4}$ Center for Astrophysics, University of Central Lancashire, Preston PR1 2HE, UK; tvcawthorne@uclan.ac.uk.

${ }^{5}$ Current address: Jodrell Bank Observatory, University of Manchester, Macclesfield, Cheshire SK11 9DL, UK; ams@jb.man.ac.uk.

${ }^{6}$ Insituto de Astrofísica de Andalucía, CSIC, Apdo. 3004, E-18080 Granada, Spain; jlgomez@iaa.es.

7 Institut d'Estudis Espacials de Catalunya/CSIC, Edif. Nexus, Gran Capita 2-4, E-08034 Barcelona, Spain; jlgomez@ieec.fcr.es.

${ }^{8}$ Department of Physics and Astronomy, Cardiff University, P.O. Box 913, Cardiff CF2 3YB, UK; Walter.Gear@astro.cf.ac.uk.
}

\section{OBSERVATIONS AND RESULTS}

We observed 3C 279 as part of a monitoring program of 15 active galactic nuclei carried out with the VLBA at $43 \mathrm{GHz}$ at 17 epochs from 1998 March 25 to 2001 April 14. We calibrated the data with the Astronomical Image Processing System (AIPS) software supplied by the National Radio Astronomy Observatory (NRAO), while we performed the imaging with the Caltech software Difmap (Shepherd 1997). The electric vector position angle (EVPA) calibration of the linear polarization was obtained by different methods: comparison with quasi-simultaneous Very Large Array (VLA) observations, comparison of "D terms" at different epochs based on the assumption that the instrumental polarization parameters change slowly with time, and the presence of EVPA-stable features in the images of the jets in $3 \mathrm{C} 279$, OJ 287, and CTA 102. The final EVPA calibration corresponds to the best agreement between the different methods and has an estimated uncertainty of $\sim 8^{\circ}$. Table 1 gives the VLBA observational parameters for each epoch. The sequence of images of 3C 279 is shown in Figure 1. The total intensity images are convolved with the average synthesized beam of $0.38 \times 0.14$ mas $^{2}$, P.A. $=-7^{\circ}$. The contours correspond to total intensity with a global peak of $17.24 \mathrm{Jy}_{\text {beam }}{ }^{-1}$ and rms noise of $9.8 \mathrm{mJy}$ beam $^{-1}$. The polarized intensity images have a global peak of $1.24 \mathrm{Jy}_{\text {beam }}{ }^{-1}$ and rms noise of $11.5 \mathrm{mJy}_{\text {beam }}{ }^{-1}$. The superposed sticks give the direction of the electric field vector.

We employed the task MODELFIT in Difmap to define the position of each component. We approximated each feature with a point-source brightness distribution and iterated to find the combinations of flux densities, positions, and circular sizes that best fit the data. We used different methods to estimate the uncertainties of the parameters: comparison of parameters obtained from different initial models, varying each parameter separately until the best $\chi^{2}$ value increases by unity, and the method suggested by Homan et al. (2001) based on the variance about the best fit of the parameterized proper-motion model. These give us the following determinations of the errors for components brighter than $0.5 \mathrm{Jy}: \sim 3 \%$ for the flux density, $\sim 0.03$ mas in right ascension $(x)$, and $\sim 0.04$ mas in declination $(y)$. The same task was used to determine the positions of polarized features using delta-function components separately for the Stokes $Q$ and $U$ images, taking the 
TABLE 1

Parameters of the VlBA Observations

\begin{tabular}{|c|c|c|c|}
\hline Epoch & $\begin{array}{l}\text { Beam } \\
\left(\operatorname{mas}^{2}\right)\end{array}$ & $\begin{array}{l}\text { P.A. } \\
\text { (deg) }\end{array}$ & Antennas \\
\hline $1998.23 \ldots \ldots \ldots \ldots$ & $0.37 \times 0.14$ & -6.0 & ALL \\
\hline $1998.41 \ldots \ldots \ldots \ldots$ & $0.38 \times 0.14$ & -5.8 & ALL \\
\hline $1998.58 \ldots \ldots \ldots \ldots$ & $0.37 \times 0.14$ & -6.4 & ALL \\
\hline $1998.76 \ldots \ldots \ldots \ldots$ & $0.37 \times 0.14$ & -6.8 & ALL \\
\hline $1998.94 \ldots \ldots \ldots \ldots$ & $0.39 \times 0.14$ & -6.0 & ALL \\
\hline $1999.12 \ldots \ldots \ldots \ldots$ & $0.39 \times 0.14$ & -8.9 & ALL \\
\hline $1999.32 \ldots \ldots \ldots \ldots$ & $0.39 \times 0.14$ & -7.8 & ALL, except NL \\
\hline $1999.55 \ldots \ldots \ldots \ldots$ & $0.40 \times 0.14$ & -8.3 & ALL \\
\hline $1999.76 \ldots \ldots \ldots \ldots$ & $0.44 \times 0.16$ & -4.6 & ALL \\
\hline $1999.93 \ldots \ldots \ldots \ldots$ & $0.39 \times 0.15$ & -12.0 & ALL, except SC \\
\hline $2000.07 \ldots \ldots \ldots \ldots$ & $0.38 \times 0.14$ & -6.0 & ALL \\
\hline $2000.26 \ldots \ldots \ldots \ldots$ & $0.37 \times 0.14$ & -7.4 & ALL \\
\hline $2000.54 \ldots \ldots \ldots \ldots$ & $0.36 \times 0.14$ & -6.0 & ALL \\
\hline $2000.75 \ldots \ldots \ldots \ldots$ & $0.38 \times 0.14$ & -6.5 & ALL, except BR \\
\hline $2000.95 \ldots \ldots \ldots \ldots$ & $0.36 \times 0.15$ & -0.6 & ALL, except LA \\
\hline $2001.08 \ldots \ldots \ldots \ldots$ & $0.40 \times 0.15$ & -5.0 & ALL, except LA, KP \\
\hline $2001.28 \ldots \ldots \ldots \ldots$ & $0.38 \times 0.14$ & -7.2 & ALL \\
\hline
\end{tabular}

average if both were present. Uncertainties in the parameters of polarized components were derived from comparison with the parameters of corresponding total intensity components.

\section{SUPERLUMINAL MOTION AND TRAJECTORIES}

We fit the $(x, y)$ position versus time for each component with the straight line that minimizes the $\chi^{2}$ statistic. This allows us to determine the mean angular speeds $\dot{x}$ and $\dot{y}$ and therefore the average proper motion $\mu=\left(\dot{x}^{2}+\dot{y}^{2}\right)^{1 / 2}$. The uncertainty of the proper motion is estimated using the $\chi^{2}$ statistic that includes both scattering of the data points around the proper-motion model and uncertainties of individual observations. We calculate the average apparent speed $\beta_{\text {app }}$ using deceleration parameter $q_{0}=0.1$ and Hubble constant $H_{0}=65 \mathrm{~km} \mathrm{~s}^{-1} \mathrm{Mpc}^{-1}$, which leads to essentially the same results (within 1\%) as a contemporary cosmology with $H_{0}=$ $70 \mathrm{~km} \mathrm{~s}^{-1} \mathrm{Mpc}^{-1}, \Omega_{M}=0.3$, and $\Omega_{\Lambda}=0.7$ for the redshift of 3C 279, $z=0.538$ (Hewitt \& Burbidge 1993).

\subsection{Motion in the Innermost Jet Region}

The $43 \mathrm{GHz}$ images from 1998 to 2001 (Fig. 1) reveal the growth of the innermost region of the jet. The emission at the western edge of the inner jet (the lowest contour corresponds

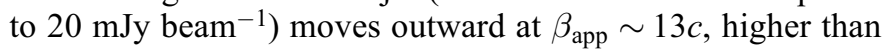
any motion observed during the previous decade (W01; J01). Figure 2 plots angular distances from the core of all total intensity components found in the inner region of the jet during our monitoring, as well as from the $43 \mathrm{GHz}$ data of W01 and J01. The identification of components corresponds to the greatest cross-epoch consistency among position angle, total and polarized flux density, EVPA, distance, and size (Fig. 2, inset).

According to W01 and J01, component C8 (B3 in J01) was ejected from the VLBI core at 1995 .6. The component ejected next is associated with C9 of W01 in 1997 March 29 and July 16, is blended with C8 on 1997 July 31 (J01) and November 16 (W01), and then continues on a trajectory defined by a proper motion $\mu=0.40 \pm 0.01$ mas $\mathrm{yr}^{-1}(12.6 \pm$ $0.3 c$ ) and ejection time $1996.89 \pm 0.04$. The path of the knot is sinuous beyond 0.5 mas from the core (Fig. 2, inset). After C9

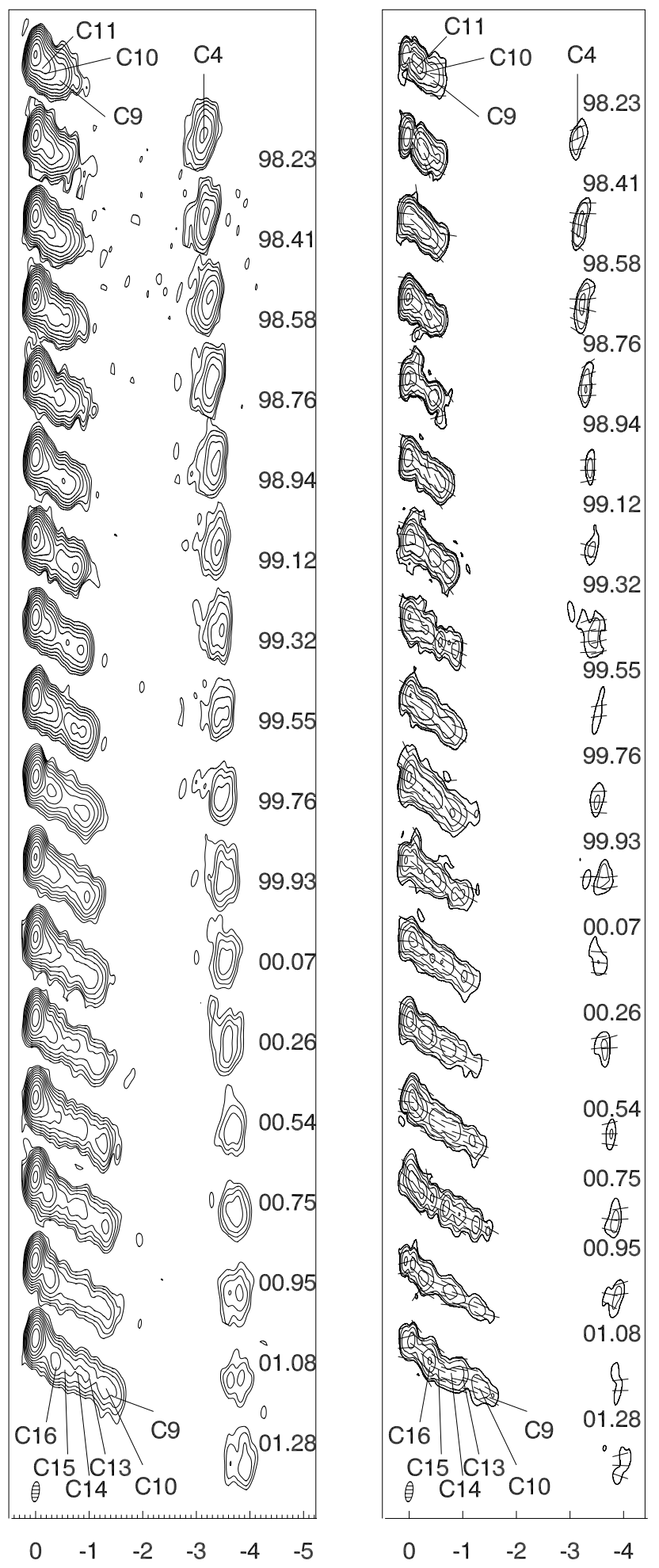

FIG. 1.-The $43 \mathrm{GHz}$ total (left) and polarized (right) intensity images of 3C 279 (see text).

a number of components are ejected with similar trajectory and apparent speed (see Figs. 1 and 2 and Table 2). This apparent speed is significantly higher than found in previous observations: for example, the velocities of knots $\mathrm{C} 5-\mathrm{C} 8$ ejected between 1990-1996 range from $4.8 \pm 0.2$ to $6.8 \pm 1.0 c$ (W01). The latter authors noted a trend that each newly ejected component has a more southern position angle than the previous one. The trend progresses in our data (see Table 2) and is easier 


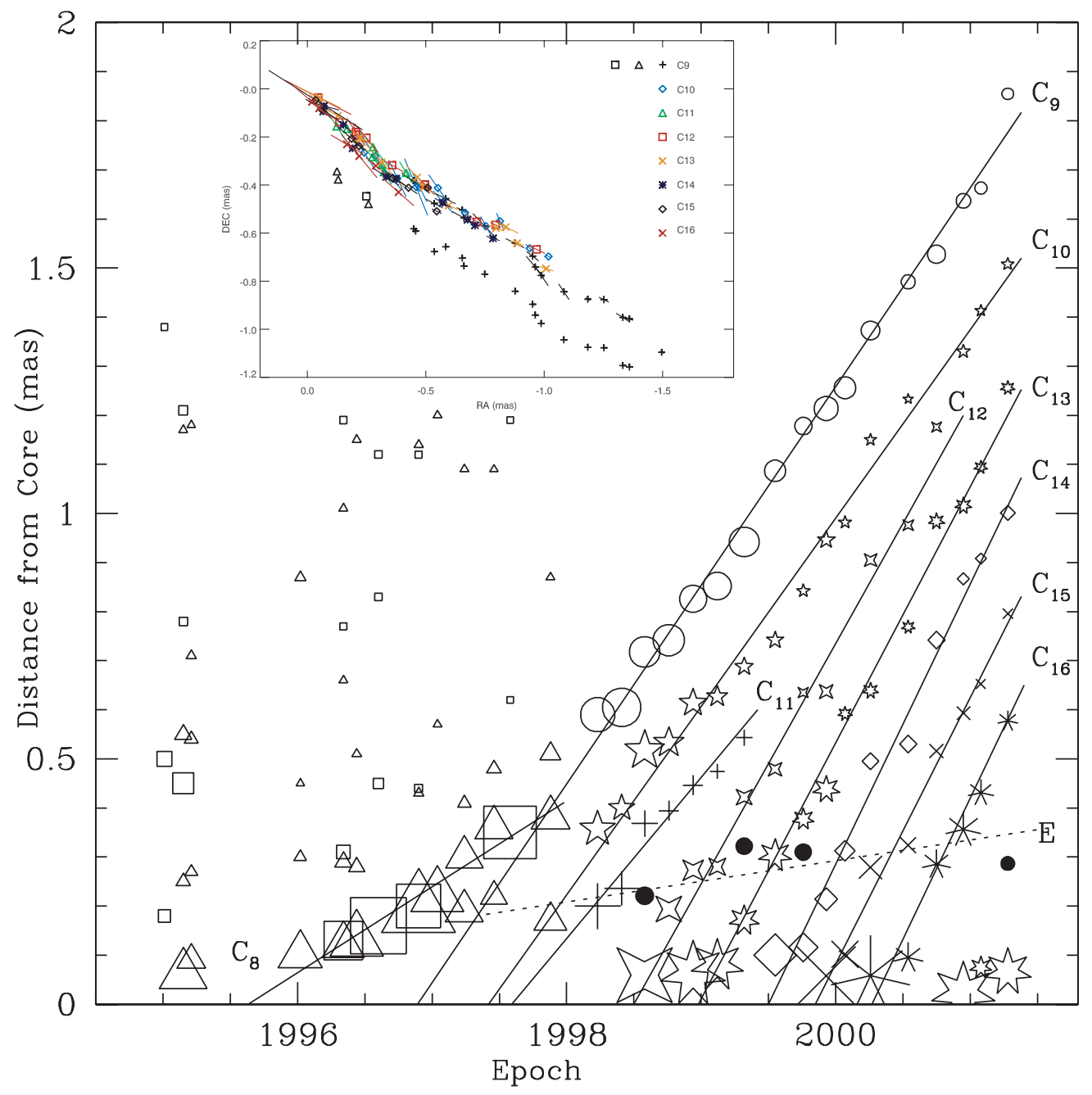

FIG. 2.-Angular distances from the core of the total intensity components from 1995 to 2001; data from 1995 to 1998 are from W01 (triangles) and J01 (squares). The sizes of the symbols are proportional to the flux densities of the respective components. Inset: Trajectories of components C9-C16 from our observations only; sticks indicate the electric vectors; the sizes of sticks are proportional to the polarized flux densities. The trajectory of knot C9 is reproduced to include the data from W01 and J01, with an offset to the south by 0.2 mas.

TABLE 2

Parameters of the Inner Jet Components

\begin{tabular}{|c|c|c|c|c|c|c|}
\hline Comp. & $\left\langle\Phi^{\circ}\right\rangle$ & $\begin{array}{c}\mu \\
\left(\text { mas yr }^{-1}\right)\end{array}$ & $\begin{array}{c}\beta_{\text {app }} \\
(c)\end{array}$ & $\left\langle\mathrm{EVPA}^{\circ}\right\rangle$ & $\left\langle m_{p}\right\rangle$ & $T_{0}$ \\
\hline $\mathrm{C} 7 \mathrm{a} \ldots \ldots \ldots \ldots \ldots$ & $-121.0 \pm 2.1$ & $0.16 \pm 0.01$ & $5.0 \pm 0.3$ & . & & 1994.67 \\
\hline $\mathrm{C} 8$ & $-130.9 \pm 2.4$ & $0.19 \pm 0.03$ & $6.0 \pm 0.9$ & -118 & 0.06 & 1995.70 \\
\hline $\mathrm{C} 9 \ldots \ldots \ldots \ldots \ldots$ & $-134.6 \pm 4.7$ & $0.39 \pm 0.03$ & $12.2 \pm 0.9$ & $-121 \pm 5$ & $0.09 \pm 0.02$ & 1996.89 \\
\hline $\mathrm{C} 10 \ldots \ldots \ldots \ldots \ldots$ & $-136.7 \pm 4.5$ & $0.27 \pm 0.05$ & $8.5 \pm 1.6$ & $-147 \pm 6$ & $0.13 \pm 0.03$ & 1997.42 \\
\hline $\mathrm{C} 11 \ldots \ldots \ldots \ldots \ldots$ & $-135.0 \pm 3.6$ & $0.32 \pm 0.05$ & $10.0 \pm 1.5$ & $-147 \pm 4$ & $0.09 \pm 0.02$ & 1997.59 \\
\hline $\mathrm{C} 12 \ldots \ldots \ldots \ldots \ldots$ & $-132.2 \pm 2.2$ & $0.42 \pm 0.03$ & $13.2 \pm 1.1$ & $-130 \pm 17$ & $0.21 \pm 0.06$ & 1998.50 \\
\hline $\mathrm{C} 13 \ldots \ldots \ldots \ldots \ldots$ & $-134.3 \pm 2.5$ & $0.47 \pm 0.03$ & $14.7 \pm 0.9$ & $-127 \pm 7$ & $0.11 \pm 0.04$ & 1998.98 \\
\hline $\mathrm{C} 14 \ldots \ldots \ldots \ldots$ & $-137.1 \pm 4.6$ & $0.55 \pm 0.04$ & $17.3 \pm 1.2$ & $-125 \pm 9$ & $0.14 \pm 0.03$ & 1999.50 \\
\hline $\mathrm{C} 15 \ldots \ldots \ldots \ldots \ldots \ldots$ & $-137.4 \pm 4.9$ & $0.54 \pm 0.03$ & $16.9 \pm 0.9$ & $-124 \pm 3$ & $0.11 \pm 0.02$ & 1999.85 \\
\hline $\mathrm{C} 16 \ldots \ldots \ldots \ldots \ldots \ldots$ & $-142.1 \pm 2.1$ & $0.53 \pm 0.05$ & $16.6 \pm 1.5$ & $-131 \pm 6$ & $0.19 \pm 0.04$ & 2000.27 \\
\hline
\end{tabular}

NoTES.-Parameters are calculated within $\sim 0.5$ mas of the core; the uncertainties correspond to the scatter of the parameter values across the epochs; data for C7a are from W01; component C10 is identified with component C9 of W01 in 1997 November 16; points of component C8 as designated by W01 but identified with C9 in the present study are not included in the computation of the parameters of C8; $m_{p}$ is fractional polarization, for C8 the polarization parameters are from Lister, Marscher, \& Gear 1998; $T_{0}$ is the time of coincidence of a component with the core, the uncertainties of $T_{0}$ are $\lesssim 0.10 \mathrm{yr}$. 


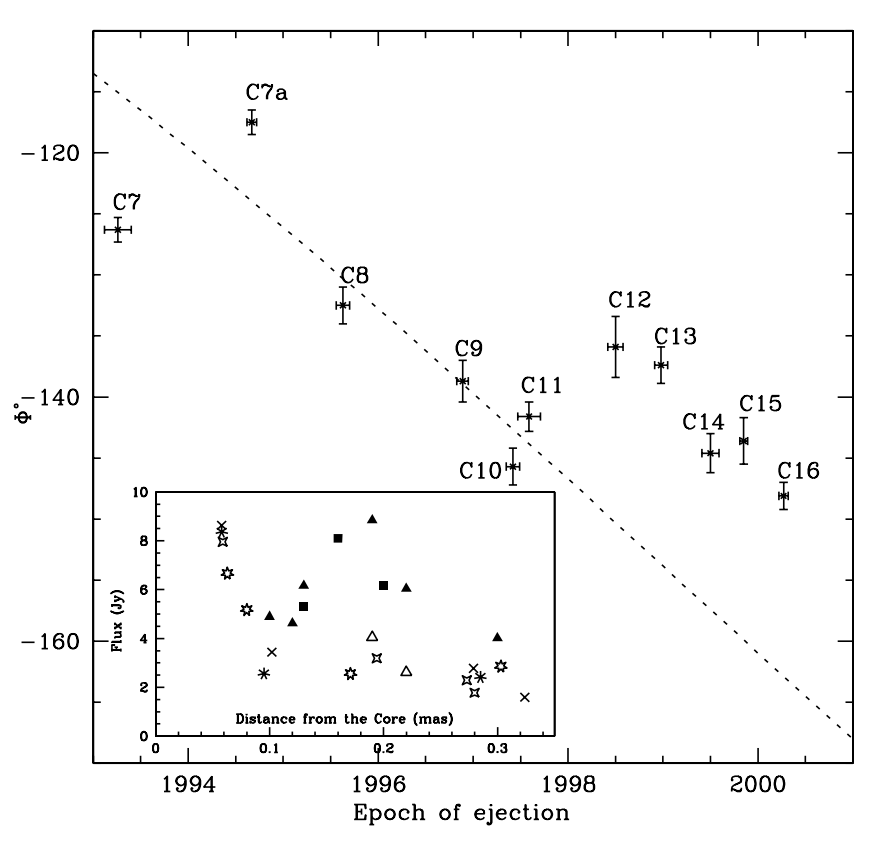

Fig. 3.-Position angle, $\Phi$, at which components C7-C16 first appear in the jet. The prediction for $\Phi$ from the precessing model by Abraham \& Carrara (1998) is shown by the dotted line. Inset: Flux density of components C8 ( filled triangles, from W01; filled squares, from J01), C9 (open triangles, from W01), C10 ( five-point stars), C12 (four-point stars), C13 (seven-point stars), $\mathrm{C} 15$ (crosses), and C16 (asterisks).

to visualize in Figure 3. This is a continuation of Figure 7 in W01, with position angles at which knots appear in the jet plotted versus ejection time. These position angles define the direction of the jet "nozzle." Figure 3 reveals a prominent single swing of the nozzle by $\sim 30^{\circ}$ to the south. Analysis of the component parameters indicates that $\mathrm{C} 9-\mathrm{C} 16$ are characterized by two common features: high apparent speed $\left(\beta_{a p p} \gtrsim\right.$ $8 c)$ and southern position angle in the jet $\left(\Phi \lesssim-131^{\circ}\right)$. In addition, the entire trajectories of components C14-C16, which move at the highest apparent speed $\left(\beta_{\text {app }} \gtrsim 15 c\right)$, have the most southern paths (see Fig. 2, inset). The projected electric vectors of components $\mathrm{C} 9-\mathrm{C} 16$ near the core align with the innermost jet direction. Although the polarization data for components ejected earlier are limited, there is a suggestion that the polarization vector follows the direction of the jet "nozzle." Table 2 also indicates the fractional polarization increases along with the proper motion.

It appears that the intrinsic viewing angle changes appreciably only when the projected position angle swings farther south than approximately $-131^{\circ}$. This is consistent with the high proper motion seen by Cotton et al. (1979) and PaulinyToth et al. (1981) in the 1970s, but is in conflict with the slow apparent speed along position angle $-135^{\circ}$ measured by Unwin et al. (1989). However, the latter might be the result of the complex long-term kinematics of the jet caused by formation of slower moving features behind the main perturbation, as found in hydrodynamical simulations (Agudo et al. 2001). Our data indicate the possible existence of slow flow in the wake of component $\mathrm{C} 8$ (see $\S 3.2$ ).

An alternative view would identify component $\mathrm{C} 9$ with $\mathrm{C} 8$, which would then need to accelerate from $6 c$ to $12.6 c$ at epoch $\sim 1997.9$ (see Fig. 2). We designate as $\mathrm{C} 8 *$ the putative accelerating component that corresponds to knot $\mathrm{C} 8$ before 1997.9 and knot C9 after 1997.9. If this version of the crossepoch identifications were correct, the trajectory of $\mathrm{C} 8^{*}$ (see

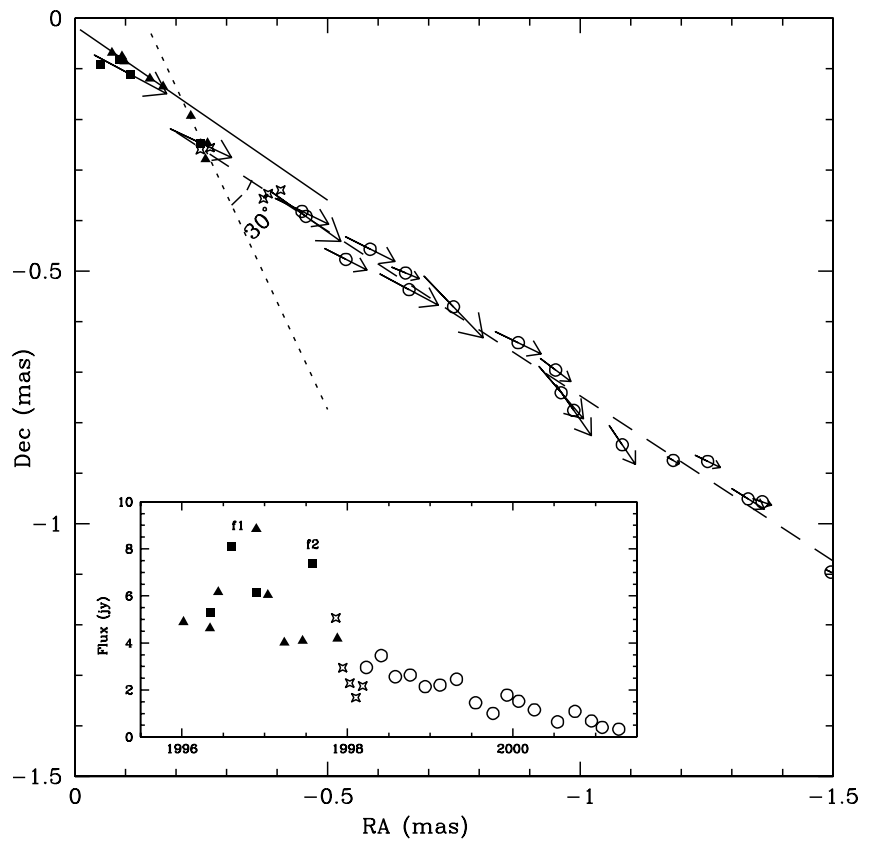

FIG. 4.-Trajectory of component C8* (see text): filled triangles are from W01, filled squares are from J01, four-point stars are from Gomez et al. (2001), where 3C 279 was used as a calibration source, and circles are from this paper; the vectors indicate the EVPAs, with the length of the vectors proportional to the polarized flux. Inset: Light curve of component $\mathrm{C} 8 *$.

Fig. 4) would be puzzling: the feature would need to move along position angle approximately $-125^{\circ}$, suddenly change the projected direction of motion by $\sim 30^{\circ}$ to the south, and then resume to within $\pm 1^{\circ}$ the previous direction of motion, but with an apparent speed $\sim 2$ times faster. It is possible in this interpretation that the core translated to the north by $\sim 0.07$ mas, since in the absence of referencing of phases to another source, all our positional information is relative to the core. However, although this would allow $\mathrm{C} 8^{*}$ to follow a path without a zig-zag in the trajectory, it would not explain the sudden acceleration. Figure 4 shows the direction of the electric vector in the component that aligns with the path. Unfortunately, during the possible zig-zag in the trajectory (1997.0-1997.5) the polarization data are absent. The light curve of $\mathrm{C}^{*}$ would contain two successive flares, $f_{1}$ and $f_{2}$ (Fig. 4, inset), just before its trajectory turned to the south. Although some of the components undergo a flare after emerging from the core, two successive flares in the same knot would be exceptional. In this light, it seems more likely that outbursts $f_{1}$ and $f_{2}$ occurred in two separate components, C8 and $\mathrm{C}$.

\subsection{Possible Slowly Moving Features}

Some of fast-moving components ejected after C8 (C9, $\mathrm{C} 11, \mathrm{C} 12$, and $\mathrm{C} 15$ ) show a delay in the motion near 0.3 mas from the core. This can be attributed to blending of the fastmoving knots and a slower feature $E$ (filled circles, Fig. 2), with apparent speed $1.3 \pm 0.4 c$. The latter may represent the feature that forms behind the brightest component $\mathrm{C} 8$ and can be interpreted as trailing shock (Agudo et al. 2001). Although, on average, ballistic motion is a good approximation for all components, the high-resolution $7 \mathrm{~mm}$ images reveal irregularities in the motion of bright knots $(\mathrm{C} 8-\mathrm{C} 14)$ inside 1 mas of the core. The separation versus time appear to accelerate and deccelerate on a timescale of a few months (see Fig. 2). Such 
complex motion of compressed features is seen in threedimensional simulations of relativistic jets, in which the threedimensional velocity vectors include components both along and transverse to the jet axis (Aloy et al. 2003).

\subsection{Motion of Component C4}

Homan et al. (2003, hereafter H03) report a change at $\sim 1998.2$ in trajectory and proper motion farther down the jet, greater than 3 mas ( $\geq 1 \mathrm{kpc}$, deprojected) from the core. Our data for $\mathrm{C} 4$ are roughly consistent with those of $\mathrm{H} 03$. There is a significant change in direction of motion $\Phi$, proper motion $\mu$, and mean flux density $\left\langle S_{\nu}\right\rangle$, from $\Phi=-112^{\circ} \pm 1^{\circ}, \mu=$ $0.23 \pm 0.03$ mas $\mathrm{yr}^{-1},\left\langle S_{\nu}\right\rangle \sim 1.3 \mathrm{Jy}$ prior to 1998.2 to $\Phi=$ $-141^{\circ} \pm 1^{\circ}, \mu=0.35 \pm 0.01$ mas $\mathrm{yr}^{-1},\left\langle S_{\nu}\right\rangle \sim 0.9$ Jy after this epoch. The event appears to occur suddenly between 1997.6 and 1998.2. The component undergoes an outburst in flux density from 1997 to 1999 before fading to a level lower than before the outburst. The apparent speed of the component

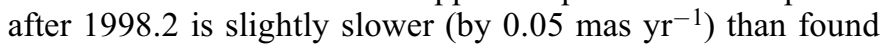
by H03. This can be explained either by differences in morphology (and therefore in brightness centroid) of the component as measured by the VLBA at 43 and $15 \mathrm{GHz}$, or by the existence of a small acceleration of the knot after 2001. Over our monitoring period from 1998.23 to 2001.28 , C4 has a fairly stable, oblique (to the jet axis) electric vector position angle of $91^{\circ} \pm 4^{\circ}$, while before 1998.2 it maintained a direction along the jet, $62^{\circ} \pm 6^{\circ}$ in 1997.58 (Marscher et al. 2002) or $\sim 65^{\circ}$ in 1996.90 (Lister, Marscher, \& Gear 1998). Since the component is optically thin $(\alpha \sim 0.9$ at $22-43 \mathrm{GHz}$; J01), this implies rotation of the magnetic vector by $\sim 28^{\circ}$ in the opposite sense to that of the trajectory change.

\section{DISCUSSION}

We observe a change in the apparent speed near the core from $5 c$ to $17 c$ and a shift in the projected position angle of component ejection from the core from $-121^{\circ}$ to $-142^{\circ}$. Figure 5 displays the dependence between (1) the proper motion $\mu$ of components and their position angle in the jet $\Phi$, (2) the proper motion and electric vector position angle of components, and (3) fractional polarization and $\Phi$ of the knots. For both parameters ( $\Phi$ and $\mu$ ) the variation exceeds the uncertainties of the individual measurements by a factor of 10 or more and the $F$-test statistic gives a probability of $99.999 \%$ that changes have occurred. Although the uncertainties in the EVPAs are considerable relative to the observed range of the $\Phi$ variations, the directions of the electric vector and jet "nozzle" are correlated (coefficient of correlation $\sim 0.7$ ), and the fractional polarization increases with proper motion. All these relations can be explained by a widening of the angle between the jet nozzle and line of sight from $\Theta \sim 0.5$ for $\beta_{\text {app }} \sim 5$ to $\Theta \leq 1.6$ for $\beta_{\text {app }} \sim 17$, with a constant Lorentz factor $\gamma \geq 20$ (see sketch in Fig. 5, right, and Fig. 4 in Homan et al. 2003). (We reject a possible solution with a decrease in viewing angle from $\sim 20^{\circ}$ to $\sim 5^{\circ}$ since this would require a consequently large change in the Doppler beaming factor, which would result in differences in flux densities of components before and after the change much greater than are observed.) If the magnetic field in the jet is transverse to the jet direction, as indicated by the polarization, the observed increase of the fractional polarization can be produced by the same geometric effect without a physical change in the magnetic field. The increase in the viewing angle produces a change in the Doppler factor from $\delta \sim 39$ to $\delta \sim 30$, which qualitatively agrees with the lower flux densities of components C9$\mathrm{C} 16$ relative to $\mathrm{C} 8$, with the lowest fluxes corresponding to the fastest and more southern-directed components C15 and C16 (see Fig. 3, inset). The higher Doppler factor could contribute to the rapid disappearance of component $\mathrm{C} 8$. H03 explain the change in projected trajectory by $\sim 26^{\circ}$ and increase in apparent speed from $8 c$ to $13 c$ of component $\mathrm{C} 4$ by an increase in the viewing angle by $\lesssim 1^{\circ}$, while the Lorentz factor remains constant at a value $\gamma \geq 15$. This reduces the Doppler factor from $\delta \geq 28$ prior to the change to $\delta \geq 23$ afterward, in agreement with the observed fading of the component as it accelerates. The viewing angle and opening angle of the jet appear to be roughly the same on parsec and kiloparsec scales, with slightly higher bulk Lorentz factor near the core.

There remains the possibility that component $\mathrm{C} 8 *$ experienced an actual acceleration (alternative view in $\S 3.1$ ). The
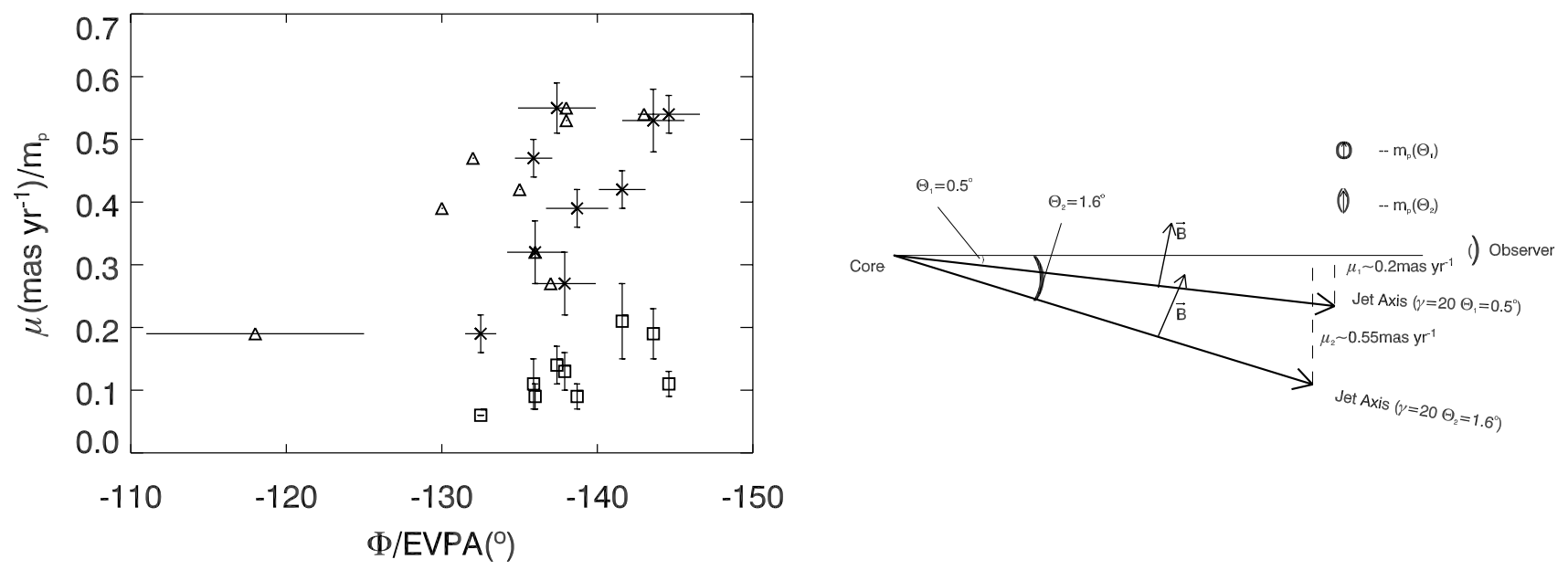

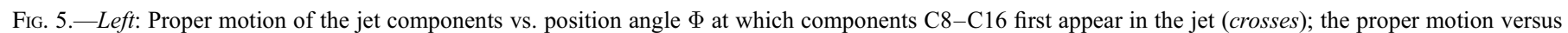

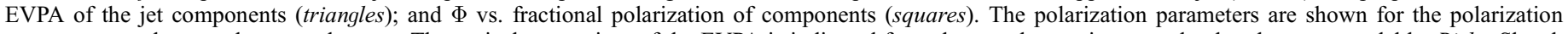

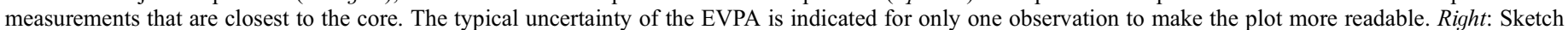

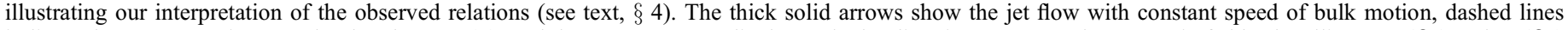

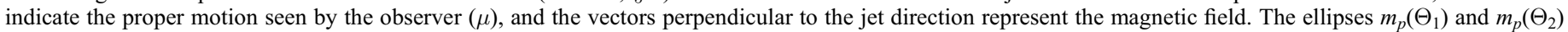
show the difference in the fractional polarization seen by the observer due to the change in position angle $\Theta$. 
change in the trajectories of $\mathrm{C} 8^{*}$ and $\mathrm{C} 4$ would then occur contemporaneously in the observer's frame. Given this temporal coincidence, it is curious that the change in projected trajectory is the same, $\sim 30^{\circ}$ to the south, and that the components attain the same apparent speed, $\sim 13 c$, after the change. One difference is that component $\mathrm{C} 8 *$ resumed its previous direction of motion but along a different (more southerly) path, while component $\mathrm{C} 4$ continued toward the new direction. This negates the possibility that the dual trajectory change was actually a shift in the position of the core. Another difference is that the electric vector of $\mathrm{C} 4$ changes in the opposite sense to the alteration in trajectory, while the EVPA of C $8^{*}$ aligns with the path both prior and after the shift in trajectory. This favors somewhat the assumption that the events are independent. If the $\mathrm{C} 4$ and $\mathrm{C} 8 *$ events were related physically, then there would need to be a "signal" (e.g., faster flow propagating through slower compressions) moving at a very high apparent velocity, $\geq 100 \mathrm{c}$. In this case the Lorentz factor of the signal would need to exceed 100, and the viewing angle would be restricted to $\lesssim 0.5$. We therefore consider such an association unlikely, but perhaps not impossible if the jet has an ultrafast spine. If there was an actual acceleration of $\mathrm{C} 8^{*}$, then it occurred at $\sim 0.5$ mas from the core, much farther than the possible location ( $\sim 0.03 \mathrm{mas})$ of the $\gamma$-ray production region in the jet $\sim 0.03$ mas (Jorstad et al. 2001b).

Abraham \& Carrara (1998) proposed a precession model of the jet "nozzle" in the quasar 3C 279 with a period of $22 \mathrm{yr}$. The general trend of the projected position angle swing follows that predicted by the model (see Fig. 3). However, the jet flow velocity $(\gamma \sim 9)$ used by Abraham \& Carrara (1998) is too low to explain the proper motions after 1997. An alter- native cause of the swing in the position angle observed by us could be magnetohydrodynamical instabilities, such as those studied by Hardee \& Rosen (1999).

Our detailed study of the kinematics of the jet of 3C 279 leads to the conclusion, suggested earlier by Lister \& Marscher (1997), that jets in objects like 3C 279 can reach Doppler factors $\gtrsim 40$, with Lorentz factors $\gtrsim 20$. Lorentz factors $\gtrsim 10$ are difficult to explain if the acceleration of the jet takes place close to the central engine, since inverse Compton energy and momentum losses would then become severe in the strong radiation environment (see Phinney 1987). However, much of the acceleration might take place well downstream, in the radio-emitting region (e.g., through magnetic forces; Vlahakis \& Königl 2004). In this case, much higher bulk Lorentz factors are possible. The extreme relativistic beaming explains why 3C 279 stands out as a quasar with high, rapidly variable flux density from radio frequencies to $\gamma$-ray energies. According to Lister \& Marscher (1997), objects like 3C 279 represent jets of modest luminosity that happen to point almost directly along the line of sight, with Lorentz factors near the high end of the range found in the general population of radio-loud active galactic nuclei.

This material is based on work supported by the National Science Foundation under grants AST 98-02941 and AST 0098579. The VLBA is a facility of the National Radio Astronomy Observatory, operated by Associated Universities, Inc., under cooperative agreement with the National Science Foundation.
Abraham, Z., \& Carrara, E. A. 1998, ApJ, 496, 172

Agudo, I., et al. 2001, ApJ, 549, L183

Aloy, M.-A., et al. 2003, ApJ, 585, L109

Cotton, W. D., et al. 1979, ApJ, 229, L115

Gómez, J. L., et al. 2001, ApJ, 561, L161

Hardee, P. E., \& Rosen, A. 1999, ApJ, 524, 650

Hewitt, A., \& Burbidge, G. 1993, ApJS, 87, 451

Homan, D. C., et al. 2003, ApJ, 589, L9 (H03) 2001, ApJ, 549, 840

Knight, C. A., et al. 1971, Science, 172, 52

Jorstad, S. G., et al. 2001a ApJS, 134, 181 (J01) . 2001b, ApJ, 556, 738

\section{REFERENCES}

Lister, M. L., \& Marscher, A. P. 1997, ApJ, 476, 572

Lister, M. L., Marscher, A. P., \& Gear, W. K. 1998, ApJ, 504, 702

Marscher, A. P., et al. 2002, ApJ, 577, 85

Pauliny-Toth, I. I. K., Preuss, E., Witzel, A., \& Graham, D. 1981, AJ, 86, 371

Phinney, E. S. 1987, in Superluminal Radio Sources, ed. J. A. Zensus \& T. J. Pearson (Cambridge: Cambridge Univ. Press), 301

Shepherd, M. C. 1997, in ASP Conf. Proc. 125, Astronomical Data Analysis Software and Systems VI, ed. G. Hunt \& H. E. Payne (San Francisco: ASP), 77

Unwin, S. C., Cohen, M. H., Biretta, J. A., Hodges, M. W., \& Zensus, J. A. 1989, ApJ, 340, 117

Vlahakis, N., \& Königl, A. 2004, ApJ, 605, 656

Wehrle, A. E., et al. 2001, ApJS, 133, 297 (W01) 